\title{
COSMIC CHEMICAL EVOLUTION
}

Edited by K. NOMOTO and J.W. TRURAN
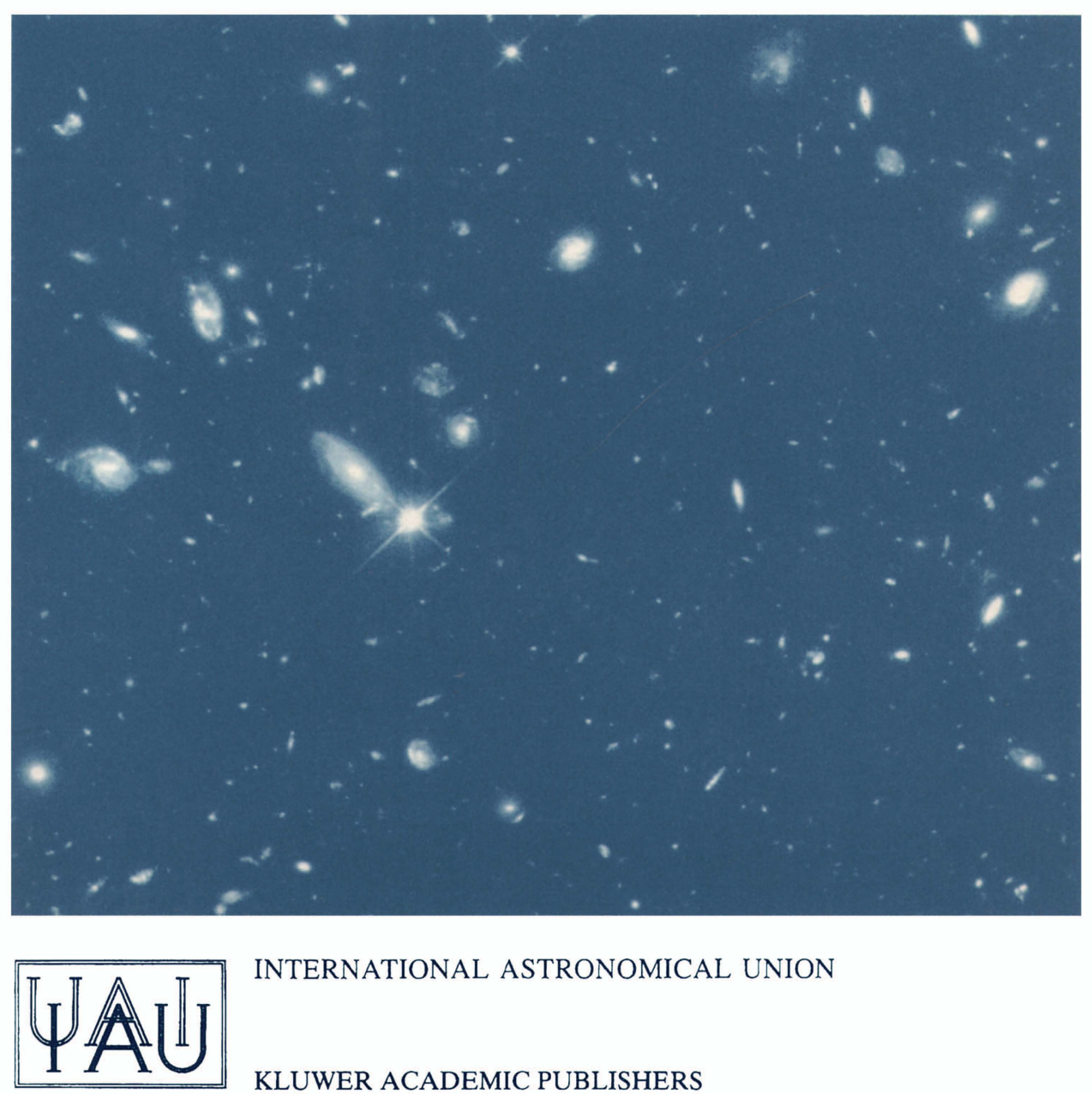

INTERNATIONAL ASTRONOMICAL UNION

KLUWER ACADEMIC PUBLISHERS 
COSMIC CHEMICAL EVOLUTION 


\title{
COSMIC CHEMICAL EVOLUTION
}

PROCEEDINGS OF THE 187TH SYMPOSIUM OF THE INTERNATIONAL ASTRONOMICAL UNION, HELD AT KYOTO, JAPAN, 26-30 AUGUST 1997

\author{
EDITED BY \\ K. NOMOTO \\ School of Science, \\ Department of Astronomy \& Research Center for the Early Universe, \\ Tokyo, Japan \\ and \\ K. W. TRURAN \\ University of Chicago \& Enrico Fermi Institute, \\ Chicago, U.S.A.
}

\section{KLUWER ACADEMIC PUBLISHERS}

DORDRECHT / BOSTON / LONDON

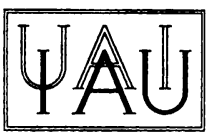


A C.I.P. Catalogue record for this book is available from the Library of Congress.

\title{
Published on behalf of the International Astronomical Union \\ by
}

Kluwer Academic Publishers, P.O. Box 17, 3300 AA Dordrecht, The Netherlands.

Sold and distributed in North, Central and South America by Kluwer Academic Publishers, 101 Philip Drive, Norwell, MA 02061, U.S.A.

In all other countries, sold and distributed by Kluwer Academic Publishers, P.O. Box 322, 3300 AH Dordrecht, The Netherlands.

\author{
Cover figure: \\ "Hubble's Deepest-Ever View of the Universe Unveils Myriad Galaxies \\ Back to the Beginning of Time \\ STScI-PRC96-01a - January 15, 1996, R. Williams and the HDF Team (ST ScI) \\ and NASA"
}

Printed on acid-free paper

All Rights Reserved

(C) 2002 International Astronomical Union

No part of the material protected by this copyright notice may be reproduced or utilized in any form or by any means, electronic or mechanical, including photocopying, recording or by any information storage and retrieval system, without written permission from the publisher.

Printed in the Netherlands. 


\section{Contents}

Preface $\quad$ xi

\section{Conference Papers in order of Presentation:}

Big Bang Nucleosynthesis

D.N. Schramm

Light Elements Abundances: New Insights on Stellar Mixing and Galactic Production

F. Primas

The First Stars

D. Arnett

Type 1A Supernovae and Chemical Evolution of Galaxies

K. Nomoto, C. Kobayashi, and H. Umeda

The Chemical Evolution of Light Elements in Our Galaxy

N. Prantzos

Nucleosynthesis in Red Giant Stars

N. Mowlavi

Fluorine Production from AGB and WR Stars

N. Mowlavi and G. Meynet

Deuterium Abundance in the Local ISM

J.L. Linsky and B.E. Wood

Abundances in Halo Population Stars

C. Sneden

Abundances in the Galactic Disk

B. Edvardsson

Abundances in a Hot Horizontal-Branch Star in the

Old Open Cluster NGC/6791

R.C. Peterson and E.M. Green 
On the Progenitors of Type Ia Supernovae

X.-D. Li and E.P.J. van den Heuvel

Abundances from $\mathrm{z}=0$ to $\mathrm{z}=5$

D.G. York

Enrichment of Heavy Elements in Clusters of Galaxies

Y. Ishimaru

On the Origin of Metallicity in Lyman-Alpha Forest Systems

M. Chiba and B.B. Nath

A Metal Enriched Dark Cluster of Galaxies at $Z=1$ M. Hattori

Are Elliptical Galaxies Really Metal-Rich?

M. Loewenstein and R.F. Mushotzky

Chemical Evolution of Spiral Galaxies from Redshift 4 to the Present

U. Fritze- v. Alvensleben, U. Lindner, and K.J. Fricke

Age Problem Based on the Abundance Ratio from a High-Redshift QSO

T. Tsujimoto, Y. Yoshii, and K. Kawara

Stellar Yields and Chemical Evolution B.K. Gibson

The Formation of First Generation Stars and Globular Clusters in Protogalactic Clouds

D.N.C. Lin and S.D. Murray

Constraints on Galaxy Formation from Extragalactic Globular Clusters

J.P. Brodie

The Evolution of Old Stellar Populations in Our Galaxy 185 S.R. Majewski 
Chemical Evolution of the Magellanic Clouds

B. Barbuy, J.A. de Freitas Pacheco, and T. Idiart

Global Evolution of the Stars, Gas, Metals, and Dust in Galaxies

S.M. Fall

Conference Summary

B.E.J. Pagel

\section{Conference Abstracts in Alphabetical Order}

Nitrogen Abundance in Oxygen-Rich Giants through

$\mathrm{K}$ to late $\mathrm{M}$

W. Aoki and T. Tsuji

Asymmetrical Delayed Detonation From a 3D

Hydrosimulation of a White Dwarf Explosion

E. Bravo ad D. García-Senz

Numerical Simulations of the Chemical Evolution of Galaxies Using Treecode-SPH

D.K. Churches, A.H. Nelson, and M.G. Edmunds

Chemical Evolution of Damped Lyman $\alpha$ Systems

K. Denda

Abundances in Metal-Rich Stars

S. Feltzing

Abundance Pattern of Supergiants in the Field of the Small Magellanic Cloud and in the Cluster NGC 330 V. Hill

Age and Metallicity Distribution of the Disk Stars from Edge-on Galaxies

A. Just

Evolution of the Colour-Magnitude Relation of Elliptical Galaxies

T. Kodama, N. Arimoto, A. Barger, and A. Aragón-Salamanca 
viii

The Optical Properties of Carbon and Graphite depend on Crystal Structure and Temperature

C. Koike, H. Shibai, T. Tanabe, H. Suto, H. Sogawa,

C. Kaito, Y. Ando, and M. Fukada

Improved Theoretical Stellar Library for Population

Synthesis Models

228

T. LeJeune and R. Buser

Chemical and Spectrophotometric Evolution of Optically Identified QSO Absorption Systems

U. Lindner, U. Fritze- v. Alvensleben, C.S. Möller, and K.J. Fricke

Comparison of Nucleosynthesis Models

O. Nakamura

The Galactic Disc Age-Metallicity Relation

231

Y.K. Ng, G. Bertelli, G. Carraro, and L. Portinari

Analysis of Elemental Carbon and Oxygen Abundances in

Carbon Stars - They have turned out to be more

Carbon-Rich than believed

K. Ohnaka and T. Tsuji

Abundances in Evolved Intermediate Mass Stars

M. Perinotto, L. Morbidelli, and P. Patriarchi

Spectral Energy Distributions of $z>2$ Galaxies in the Hubble Deep Field

M. Sawicki and H.K.C. Yee

Blue 'Elliptical' Galaxies in the Hubble Deep Field

Y. Shioya and T. Yamada

The Symbiotic-Barium Stars Link

V.V. Smith, K. Cunha, A. Jorissen, and H.M.J. Boffin

The Spectral Evolution of Elliptical Galaxies in FIR and beyond

T. Takagi and N. Arimoto 
Nucleosynthesis in ONEMG Novae: Comparison with Observations

S. Wanajo, K. Nomoto, and M. Hashimoto

Constraints on Cosmic Chemical Evolution from the Gamma-Ray Background

K. Watanabe, D.H. Hartmann, and M.D. Leising

Disk-Wind Models for Broad Absorption Line QSO's

T.M. Yamamoto

Supernova Observing Web (SNOW) Project with the Public Astronomical Observatories in Japan

H. Yamaoka, M. Doi, T. Shigeyama, N. Yasuda, and $M$. Watanabe

Element Abundances in Metal-Poor Stars

G. Zhao and Y.Q. Chen 